\title{
Occlusal caries: wherefore art thou?
}

\author{
Occlusal caries detection in primary teeth: a comparison of DIAGNOdent with conventional methods by D. C. Attrill \\ and P. F. Ashley BrDent J 2001; 190: 440-443
}

\section{Objective}

To compare the accuracy and repeatability of three diagnostic systems (DIAGNOdent, visual and radiographic) for occlusal caries diagnosis in primary molars.

\section{Design}

Two examiner, in vitro, blinded study. Histological gold standard.

\section{Materials and methods}

58 occlusal surfaces of primary molars examined in turn by two examiners using each of three diagnostic systems (DIAGNOdent, visual and radiographic). These results were compared with a histological gold standard. Sensitivity and specificity were calculated for each diagnostic system at a range of thresholds. Inter- and intra- examiner repeatability were calculated for each diagnostic system using the kappa statistic.

\section{Main outcome measures}

Sensitivity, specificity, inter and intra examiner repeatability for each diagnostic system.

\section{Results}

The highest sensitivity values were provided by DIAGNOdent ( 0.77 and 0.80 , examiners 1 and 2 respectively) however this was offset by a lower specificity $(0.82$ and 0.85$)$ than all other systems with the exception of examiner 1 at $\mathrm{V} 1$ visual threshold. The

DIAGNOdent gave the highest values of kappa for intra- and inter- examiner repeatability with the exception of intra-examiner repeatability for examiner 2 where visual diagnosis had the highest value of kappa.

\section{Conclusion}

The DIAGNOdent was the most accurate system tested for the detection of occlusal dentine caries in primary molars. The performance of the DIAGNOdent systems was not statistically significantly better than that achieved using visual examination for non-cavitated teeth (V1 and V2 threshold). DIAGNOdent may prove useful as a predictive clinical tool, however with appropriate training visual examination may offer similar results without the need for additional equipment.
In Brief
- This paper investigates three methods for the detection of early occlusal carious lesions in primary teeth
- A new laser-based diagnostic system (DIAGNOdent) was shown to offer the best combination of predictive capabilities (sensitivity and specificity) when compared with visual and radiographic
- Visual examination remains a powerful predictor of caries
- DIAGNOdent may be particularly useful in the monitoring of lesion progression, or as part of a screening process

\section{Comment}

The accurate diagnosis of dental caries 1 has been a problem of great interest to the dental profession, those financing dental services and to journalists seeking a story. Elderton in his studies in Dundee highlighted the lack of consistency between dentists when planning restorative interventions. ${ }^{1}$ As a caring health profession we must be sure that the placement or removal of restorations because of dental caries is fully justified; as intervention for no sound clinical reason is uncomfortable for the patient and wastes scarce financial resources.

The importance of a scientific approach to caries diagnosis was highlighted by the British Postgraduate Medical Federation video 'From Black so White' which raised three key issues, namely: ${ }^{2}$

- Are you aware that probes can damage teeth?

- Do you know about remineralisation?

- Do you know enough about the 'sealant restoration'?

I suspect that, despite all the evidence, ${ }^{3,4}$ a huge number of dentists in 2001 will grasp a probe and attack sound enamel in an attempt to find caries. I just wish that all members of the profession would place enamel on a pedestal and treat it with respect. It is far and away the best restora- tive material we have. It copes with changes in temperature, acid attack and it looks good. In addition, it will remineralise so why do we attack it with such fren$\mathrm{zy}$ - are we so worried we may miss caries? If yes, there are other ways to look for this insidious enemy, the simplest being a bright light and a dry tooth, hardly expensive or high tech!

Astrill and Ashley in their paper have looked at occlusal caries diagnosis in primary teeth using a laser-based system called the DIAGNOdent, comparing it with visual and radiographic diagnostic techniques. The study is elegantly undertaken, the results clearly presented and they certainly show the potential value of the DIAGNOdent system when diagnosing occlusal caries. Interestingly the authors noted that bitewing radiography performed poorly. However in the conclusion they highlight the fact that a visual examination offers similar results to the DIAGNOdent without the need for expensive equipment. They did stress however that the visual system did require appropriate training, but no advice was offered on what the training requirements would be.

Problems with caries diagnosis in primary teeth relate mainly to the approximal surfaces which are difficult to see because of the wide contact points and this is where bitewing radiographs can be of great value, so we should not dismiss the use of radiography when taking a holistic view of caries diagnosis in the young child. However indiscriminate use of bitewing radiography is difficult to justify unless a visual examination suggests further tests are required to make a diagnosis of caries.

Attrill and Ashley noted that a simple visual examination when used in the appropriate manner is the yardstick against which other diagnostic systems must be compared and unless a new technique is much better we as a profession should continue to use our eyes, drying air and a bright light.

\section{Anthony S. Blinkhorn}

Professor of Oral Health, University of Manchester

1 Elderton R I, Nuttall N M. Variation among dentists in planning treatment. Br Dent J 1983; 154: 201-206.

2 From Black to White. Dental progress: A DHSS section 63 initiative. London: British Postgraduate Medical Federation, University of London, UK.

3 Rock W P. The diagnosis of early carious lesions: A review. J Paediatr Dent 1987; 3: 1-6.

4 Tveit A B, Espelid I, Fjelltveit A. Clinical diagnosis of occlusal dentine caries. Caries Res 1994; 28: 368-372. 